\title{
SISTEMA DE GESTIÓN DE CALIDAD EN UNA UNIVERSIDAD PÚBLICA PERUANA: ANÁLISIS DE LOS PROCESOS PRINCIPALES Y GESTIÓN DE RIESGOS
}

\section{RESUMEN}

Se presenta un análisis del sistema de gestión de la calidad de la Facultad de Ciencias de una universidad pública en el Perú. El objetivo es la identificación, la descripción, la caracterización y el análisis de riesgos de procesos principales de la gestión universitaria. Metodológicamente se usó la técnica de revisión documentaria para efectos del diagnóstico, además se usó la NORMA ISO 9001:2015 como guía para identificar, caracterizar procesos, y hacer un análisis de riesgos según el enfoque a dichos procesos. Los resultados permitieron identificar y describir los procedimientos y en base a ellos realizar una propuesta para la gestión de riesgos de los procesos más importantes en el marco de la implementación de un sistema de gestión de calidad.

PALABRAS CLAVE

Enfoque en procesos, sistema de gestión de la calidad, análisis de riesgos, caracterización de procesos, gestión universitaria.

\section{ABSTRACT}

An analysis of the quality management system of the Faculty of Sciences of a public university in Peru is presented. The objective was the identification, description, characterization and analysis of risks of the main processes of university management. Methodologically, the document review technique was used for diagnosis purposes, in addition, the ISO 9001:2015 STANDARD was used as a guide to identify, characterize processes, and carry out a risk analysis according to the process approach. The results made it possible to identify and describe the procedures and make a proposal for risk management of the main processes within the framework of the implementation of a quality management system.

KEYWORDS

Approach to processes, quality management system, risk analysis, characterization of processes, university management.
QUALITY MANAGEMENT SYSTEM IN A PERUVIAN PUBLIC UNIVERSITY: ANALYSIS OF THE MAIN PROCESSES AND RISK MANAGEMENT

Ana María Adriazola León

ORCID: 0000-0002-1369-4027

amadrile@unife.edu.pe

Diego Masías Jara Sánchez

ORCID: 0000-0002-6603-8496

psidiego20@gmail.com

Isabel Mercedes Lara Torres

ORCID: 0000-0001-9156-2292

chavela1lara8@hotmail.com

Gisella Liliana Matos Cuzcano

ORCID: 0000-0001-5630-3746

gisella_matos@yahoo.com

Patricia Linda Reyes Sánchez

ORCID: 0000-0002-6603-8496

20101307@lamolina.edu.pe

\section{Introducción}

$\mathrm{E}$ n el Perú, en el último decenio se han hecho grandes esfuerzos para garantizar las condiciones básicas de calidad y acreditar la calidad del servicio universitario, para tal fin desde el 2006 existe el Sistema Nacional de Evaluación, Acreditación y Certificación de la Calidad Educativa - SINEACE.

Este organismo adscrito al Ministerio de Educación del Perú se encarga del proceso de acreditación que garantiza la calidad educativa de las universidades y de los programas profesionales de estudio. También se han desarrollado diversas investigaciones en el Perú; tales como la de Yamada, Castro, Bacigalupo \& Velarde (2013) y Yamada (2013) en el que se 
señala la urgencia de contar con universidades de calidad que garanticen el egreso de profesionales que necesita el Perú para desarrollarse, además reportan que los estudiantes ingresan a las universidades con un bajo nivel de habilidades cognitivas producto de las deficiencias en la educación primaria y secundaria.

La calidad educativa para el SINEACE (2016) es el nivel óptimo formativo que se debe alcanzar en la institución educativa, esto implica ser eficientes y eficaces para brindar este servicio; de tal manera, que cubra con todas las expectativas del estudiante y de la demanda social que espera un profesional competente.

Para garantizar la calidad en una organización educativa es importante la aplicación del enfoque basado en procesos, este implica tener una visión que permita observar el conjunto de actividades vinculadas entre sí, analizando sus interacciones para alcanzar los resultados organizacionales.

Como referencia para el presente estudio se ha considerado la norma ISO 9001:2015, este documento es vital para identificar los procesos en la universidad, tener claridad sobre ellos y no necesariamente para certificar la universidad con una ISO.

Dichos documentos presentan un conjunto de normas y principios sobre calidad y está explicado de forma clara y sencilla qué debe hacer cada institución para contar con un Sistema de Gestión de la Calidad - SGC implementado y así poder certificarse; la ISO 21001 es una norma del 2018 que es, específicamente, para organizaciones educativas, está muy vinculada con la norma anterior.

Diversos estudios demuestran que la norma ISO 9001:2015 se está implementado en las universidades para optimizar la calidad del servicio (Dolzhanskyy et al., 2016; Hernandez et al., 2017; Negrete et al., 2020; Zaher, 2020). Es necesario recalcar que la calidad es solo un medio para optimizar el servicio, el fin de la universidad debe ser la formación integral de la personalidad de los futuros profesionales (Ortiz, 2008).

La Universidad de estudio es de carácter público y nació el año 1901 en el Perú, actualmente tiene como objetivo acreditar sus programas de estudio en base a los estándares de calidad señalados en el "Modelo de Acreditación para Programas de Estudios de Educación Superior Universitaria" del SINEACE (2016) en el Perú. Para el caso de estudio se eligió a la Facultad de Ciencias que cuenta con los programas profesionales de: Biología, Ingeniería Ambiental y Meteorología, de los cuales Biología se encuentra acreditada bajo los estándares del primer modelo de acreditación (CONEAU, 2009) desde el 2017 por un período de tres años, en este no se exigía un SGC implementado. Es en este marco, que la Facultad se encuentra diseñando un SGC con un enfoque basado en procesos, puesto que esta implementación es una necesidad y requisito que ha planteado el SINEACE para las posteriores acreditaciones.

Para este caso de estudio se formuló la siguiente pregunta: ¿Cuáles son los procesos de la Facultad de Ciencias de la Universidad? Y se establecieron los siguientes objetivos: a) Identificar los procesos y elaborar los mapas de nivel 0 y 1 considerando sus interrelaciones, b) caracterizar los procesos misionales y c) realizar el análisis de los riesgos de los procesos misionales, ya que no se evidenció una gestión de procesos en dicha Facultad.

\section{Marco Teórico}

\section{Calidad educativa}

Existen diversas definiciones sobre la calidad educativa, Bermeo (2011) desarrolló un estudio sobre la calidad universitaria donde señala que hay diversos planteamientos que se vinculan incluso con la noción de calidad total en la educación y que estos conceptos están vinculados con la efectividad en la gestión para lograr los objetivos. Illescas, Cabezas \& 
Diener (2006) señalan que la calidad debe ser demostrada por los docentes desde su proceso de incorporación, desarrollo y nombramiento; en los estudiantes desde su ingreso, evaluación, becas y reconocimiento; en la investigación producida; en los planes y cursos de estudio y el personal administrativo que acompaña a los docentes.

El SINEACE (2016) indica que "la calidad educativa es el nivel óptimo de formación que deben alcanzar las personas para enfrentar los retos del desarrollo humano, ejercer su ciudadanía y continuar aprendiendo durante toda la vida". Esto deja entender que el proceso formativo universitario debe ser eficaz tomando en cuenta las necesidades de los futuros profesionales. Esta es la definición que se asume para el presente estudio.

\section{Sistema de Gestión de la Calidad}

Está definido como el conjunto de partes que conforman el programa académico o la organización, estos se encuentran interrelacionados e interactúan estableciendo políticas, objetivos y procesos que permiten alcanzar las metas institucionales vinculadas con la calidad (SINEACE, 2016).

El SINEACE como parte de los estándares de calidad para la acreditación institucional y de programas de estudios de educación superior universitaria exige un sistema de gestión de la calidad implementado. Al implementarse generará varios beneficios como la generación de mayor eficiencia, mejora de los procesos y la comunicación interna, entre otros aspectos que favorecen la gestión universitaria.

La norma ISO 9001:2015 permite la certificación de las organizaciones que cumplen con los requisitos vinculados al SGC. La norma ISO 21001:2018, está basada en la norma anterior, sin embargo, es más específica para organizaciones educacionales, en esta se establecen los requisitos para el cumplimiento del sistema de gestión de las organizaciones educativas.

\section{Enfoque en procesos}

Este “implica la definición y gestión sistemática de los procesos y sus interacciones, con el fin de alcanzar los resultados previstos de acuerdo con la política de la calidad y la dirección estratégica de la organización" (ISO 9001:2015, 8). Establecer los procesos y sus interrelaciones es vital para cumplir con altos estándares de gestión en la universidad.

El enfoque de procesos permite establecer el mapa de procesos. Este es un gráfico que abarca los procesos de una organización, de tal forma que se observe el funcionamiento y desempeño de estos, aporta claridad para el desarrollo del plan estratégico. Por ello, en este estudio se busca identificar los procesos estratégicos (vinculados a la visión, planificación global, las funciones vitales de la gerencia); principales (misionales o que corresponden al objetivo vital que es formar profesionales competentes); de soporte (o de apoyo como los servicios psicopedagógicos y otros).

\section{Caracterización del proceso}

Es el análisis profundo de cada proceso, en la caracterización se establecen los elementos de entrada y salida, además se identifica el objetivo del proceso, responsables, partes interesadas, registros de control, indicadores de la gestión y descripción de actividades (Agudelo \& Escobar, 2010).

Desarrollarla, permite estudiar si este proceso se puede agilizar o automatizar, detectar riesgos, observar pasos que no se estén desarrollando a cabalidad e incluir nuevos indicadores, si fuese necesario.

\section{Análisis de riesgos}

Es el procedimiento para la identificación de obstáculos producto de la incertidumbre, que permiten establecer oportunidades, de tal forma que se pueda tomar decisiones para afrontarlas de manera adecuada (ISO 9001:2015). 
Este es un punto muy importante en la ISO 9001:2015 puesto que hay información sobre el pensamiento basado en riesgos para la organización, incluso es uno de los requisitos el desarrollo del análisis de riesgos.

\section{Metodología}

\section{Diagnóstico de la institución educativa}

Para el desarrollo del diagnóstico de la Facultad de Ciencias se utilizó una metodología cualitativa. Los métodos cualitativos según Ruíz: "Son los que enfatizan conocer la realidad desde una perspectiva de la persona enterada, experimentada..." (2012, p.17), de esta forma se puede comprender el significado especial de un fenómeno desde el mismo protagonista. Para el estudio se desarrolló la recolección de la documentación necesaria, y a través de la técnica de revisión documentaria y observación, se llevó a cabo el estudio y análisis de los documentos.

\section{Identificación y caracterización de procesos}

Los procesos se identificaron mediante el análisis de actividades que se desarrollan en la Facultad, se configuraron en un mapa de procesos de nivel 0 (es el mapa más general) y se clasificaron de acuerdo con su importancia para la facultad en procesos: estratégicos, principales y de soporte. La caracterización consistió en la definición del objetivo, el alcance de cada proceso y la identificación de las entradas, salidas, controles (indicadores) y recursos.

\section{Análisis de riesgos}

Según la Norma ISO 9001:2015 para la identificación y análisis de riesgos se utilizó el método de Análisis de Modos de Fallo y Efectos (FMEA), este se desarrolla durante el diseño de un producto o proceso. Este método consiste en la identificación de los problemas o eventos posibles que pudieran surgir en los productos o procesos capaces de crear impactos positivos o negativos, y decidir qué hacer al respecto para alcanzar los objetivos propuestos (ISO 9001:2015).

Para el presente trabajo los pasos que se han seguido, en referencia al método FMEA, fueron: i) identificación de los riesgos; ii) determinación de la probabilidad de ocurrencia; iii) clasificación de los riesgos, ver si son aceptables o inaceptables, y luego obtener el valor de riesgo y, iv) determinación de las acciones o establecimiento del plan de mejora.

\section{Resultados}

\section{Diagnóstico}

Al realizar el análisis del contexto se observó que, uno de los problemas principales es que la Facultad de Ciencias, aún no tiene identificados sus procesos; por tanto, no se han realizado las caracterizaciones, ni el análisis de los riesgos, lo que impide tomar acciones que mitiguen los riesgos que se presenten durante el desarrollo de los mismos.

\section{Mapa de procesos}

Un mapa de procesos permite identificar cada proceso que se realiza dentro de una institución; asimismo, determinar las interacciones que se presentan entre ellos, de manera que se les pueda optimizar.

En el presente estudio se desarrolló el mapa de procesos. En la figura 1 se muestra el mapa de procesos de la Facultad de Ciencias de la Universidad Nacional de estudio de nivel 0 y sus interrelaciones. En este se observa claramente los procesos vinculados a la gestión estratégica, los procesos principales relacionados con la gestión institucional ligados de forma directa al desarrollo del servicio educativo y los procesos de soporte referidos a la gestión del soporte, tales como los servicios de bienestar estudiantil y recursos tecnológicos, entre otros. 


\section{Figura 1}

Mapa de procesos de nivel 0
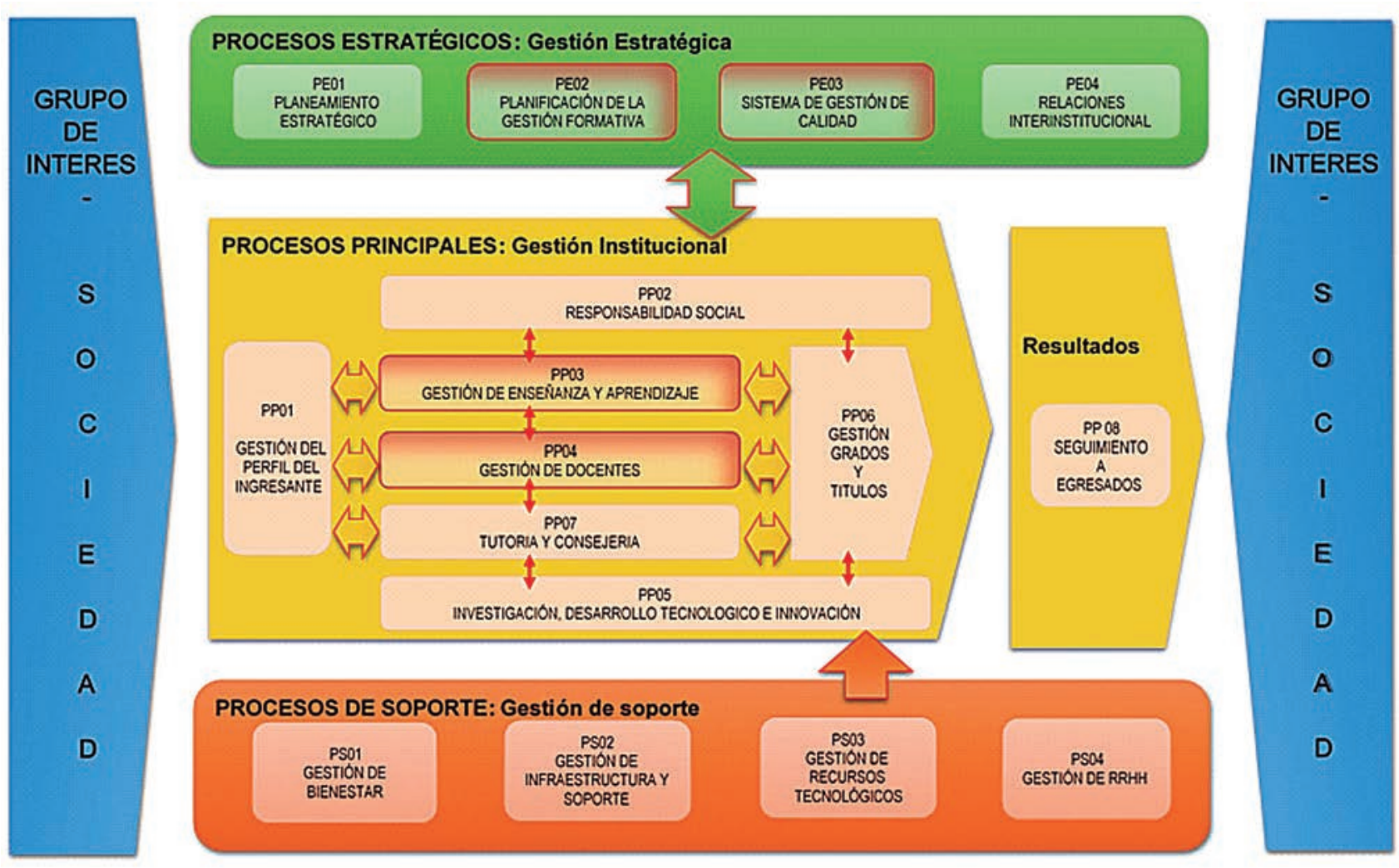

Fuente: Elaboración propia.

Para los macro procesos de planificación de la gestión formativa, sistema de gestión de calidad, gestión de enseñanza y aprendizaje, y gestión de docentes se elaboraron los mapas de procesos de nivel 1 .

Dentro de los procesos principales, se encuentra el proceso denominado Gestión de Enseñanza y
Aprendizaje. En la figura 2 se observa el mapa de nivel 1 de dicho proceso, este es muy importante dentro de la universidad, se muestra claramente que contiene siete procesos del nivel 1, las flechas indican las interrelaciones y se observa que se vincula con el proceso denominado Tutoría y Consejería. 


\section{Figura 2}

Mapa de proceso de gestión de enseñanza y aprendizaje (nivel 1)

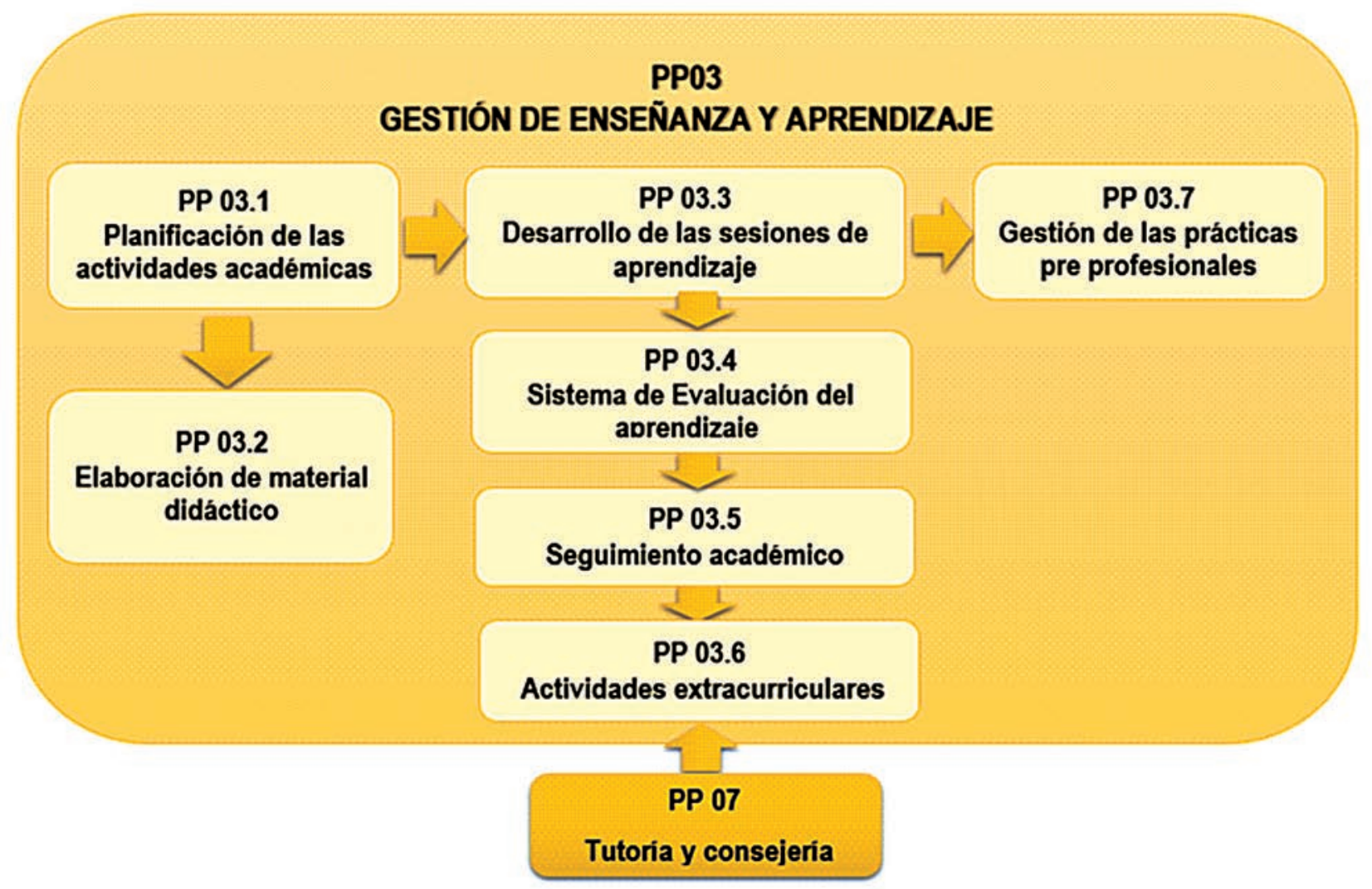

Fuente: Elaboración propia.

\section{Caracterización de procesos}

Se caracterizaron 7 procesos:

- Gestión del perfil del ingresante (PP 01).

- Planificación de las actividades académicas (PP 03.1).

- Sistema de evaluación del aprendizaje (PP 03.4).

- Selección y contratación docente (PP 04.1).
- Evaluación del desempeño docente (PP 04.2).

- Gestión de grados y títulos (PP 06).

- Tutoría y consejería (PP 07).

En la figura 3 se observa la caracterización del proceso denominado Sistema de evaluación del aprendizaje, en esta se identificó el objetivo de este sistema, el alcance, las entradas y salidas del proceso, así como los indicadores y los recursos que se necesitan para la ejecución del proceso. 


\section{Figura 3}

Caracterización del proceso: Sistema de evaluación del aprendizaje

\section{CARACTERIZACIÓN DEL PROCESO}

\section{SISTEMA DE EVALUACIÓN DEL APRENDIZAJE (PP 03.1}

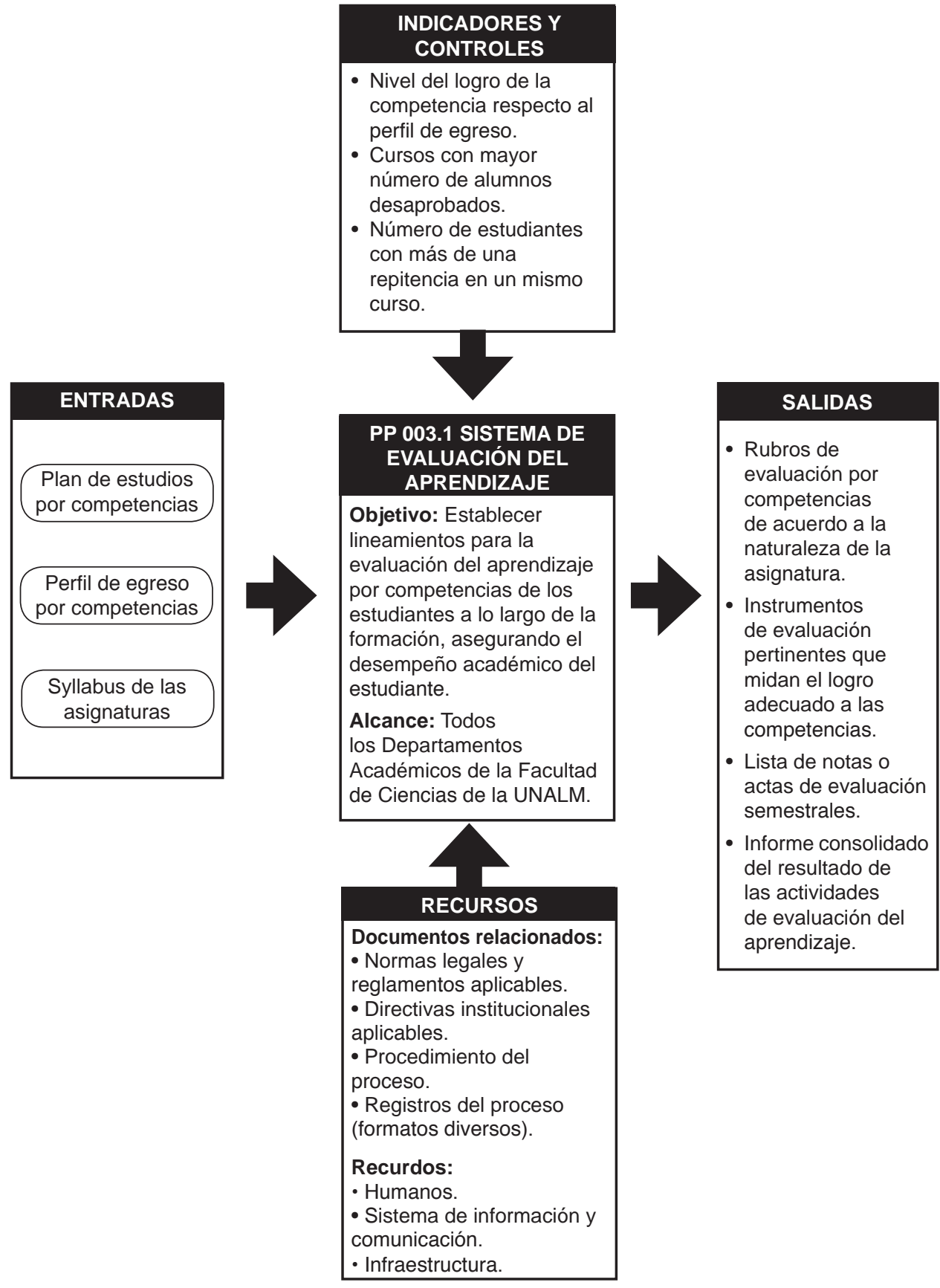

Fuente: Elaboración propia.

También se muestra la caracterización del proceso denominado Evaluación del Desempeño Docente, este es muy importante puesto que es la imagen de la universidad frente a los estudiantes y además es el docente quien materializa los objetivos de la carrera y la universidad, así se podrá observar cómo está el desempeño docente para poder tomar acciones de fortalecimiento o desarrollar competencias para mejorar la enseñanza. 


\section{Figura 4}

Caracterización del proceso: Evaluación del desempeño docente

\section{EVALUACIÓN DEL DESEMPEÑO DOCENTE PP 04.2}

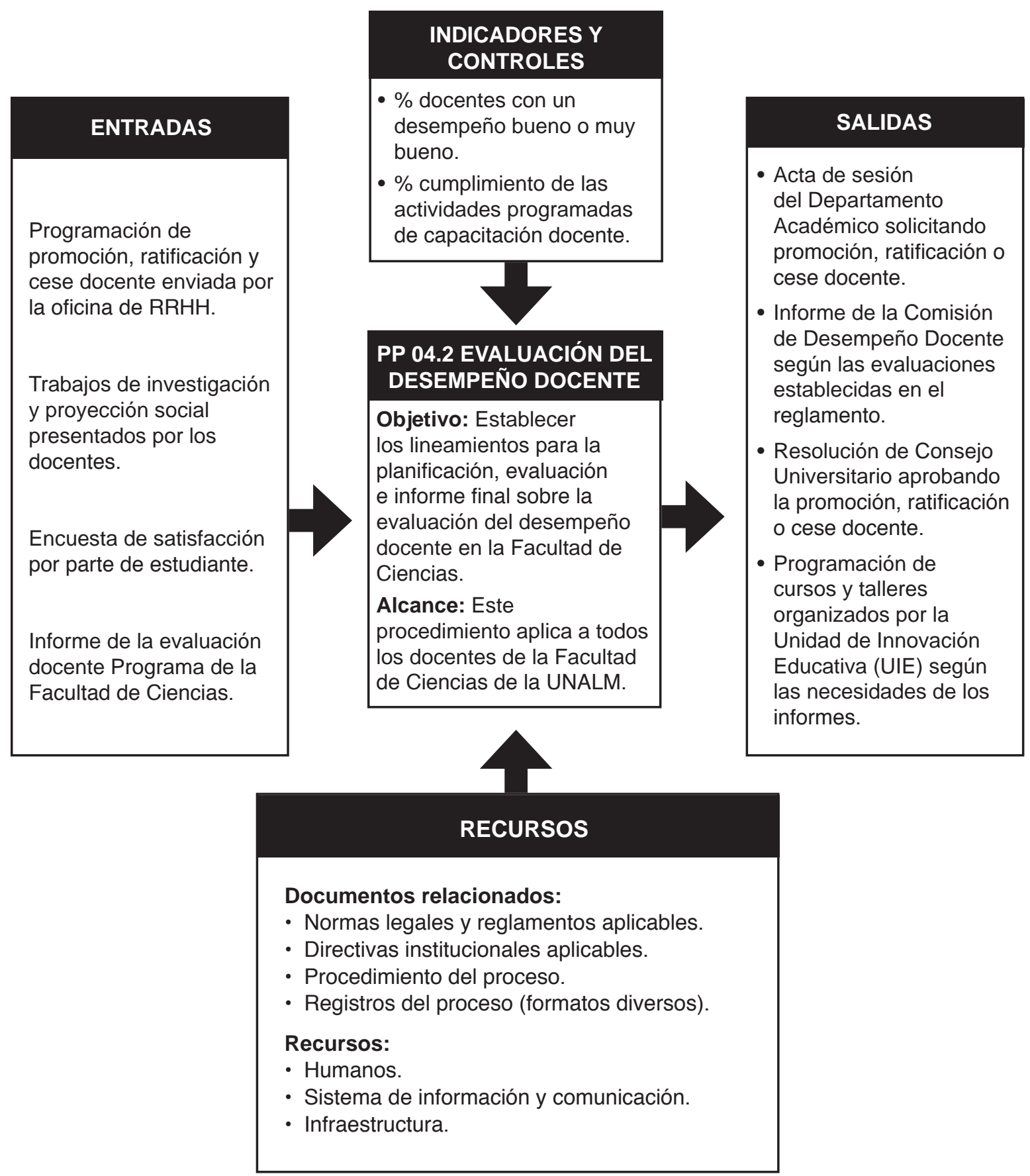

Fuente: Elaboración propia. 


\section{Análisis de riesgos}

Según la ISO 9001:2015, tener identificados los riesgos permite reconocer las causas por las que los procesos y el SGC se desvían de los objetivos planificados por la organización; por ello, el pensamiento basado en riesgos permite reducir los efectos negativos y aprovechar la oportunidad que genera esto. Es en este ámbito que se identificaron los posibles riesgos de los siete procesos caracterizados.
El impacto y, por tanto, el tipo de tratamiento de un riesgo del producto/servicio no conforme se determinó según la siguiente matriz de la tabla 1. En esta se observa que el riesgo se evalúa tomando en cuenta la probabilidad y severidad de sus efectos en la organización, esto determina un puntaje al cual le corresponden formas de tratamiento (ver Tabla 1).

\section{Tabla 1}

Matriz de riesgos. Establecimiento de criterios de los riesgos

\begin{tabular}{|c|c|c|c|c|}
\hline \multirow[b]{2}{*}{ PROBABILIDAD } & \multicolumn{3}{|c|}{ SEVERIDAD } & \multirow[t]{2}{*}{ TRATAMIENTO } \\
\hline & Baja (1) & Media (2) & Alta (3) & \\
\hline Baja (1) & 1 & 2 & 3 & $\leq 2$ : Concesión, liberación. \\
\hline Media (2) & 2 & 4 & 6 & $\begin{array}{l}>2 \text { y }<6 \text { : Reproceso, reclasificación, } \\
\text { reparación, permiso de desviación. }\end{array}$ \\
\hline Alta (3) & 3 & 6 & 9 & $\geq 6$ : Acción correctiva, desecho. \\
\hline
\end{tabular}

Fuente: ISO 9001:2015

La Tabla 2 muestra el análisis de riesgo del proceso: Sistema de Evaluación del Aprendizaje. En esta se determinó los posibles riesgos que se pueden presentar durante el desarrollo del proceso, se estableció la probabilidad de ocurrencia y la severidad, de manera que se halle el impacto, así como el tratamiento a seguir para mitigar el riesgo.
La identificación de procesos, caracterización y análisis de riesgo hacen que la universidad facilite su proceso de implementación de un SGC, el cual permite la efectividad de la gestión y además el logro de uno de los estándares de calidad del SINEACE para la acreditación. Por este motivo, los resultados se deben difundir en la comunidad universitaria. 


\section{Tabla 2}

Análisis de riesgo del proceso Sistema de evaluación del aprendizaje

\begin{tabular}{|c|c|c|c|c|c|c|}
\hline $\mathrm{N}^{\circ}$ & RIESGO & $\begin{array}{l}\text { PROBABILIDAD } \\
\text { (1=Baja, } 2=\text { media, } \\
3=\text { alta })\end{array}$ & $\begin{array}{l}\text { SEVERIDAD } \\
\text { (1=Baja, } 2=\text { media, } \\
3=\text { alta })\end{array}$ & IMPACTO & TRATAMIENTO & $\begin{array}{l}\text { SUSTENTO DEL } \\
\text { TRATAMIENTO }\end{array}$ \\
\hline 1 & $\begin{array}{l}\text { Los criterios } \\
\text { de evaluación } \\
\text { del sílabo } \\
\text { no miden el } \\
\text { logro de las } \\
\text { competencias } \\
\text { del curso. }\end{array}$ & 1 & 3 & 3 & $\begin{array}{l}\text { - Rediseño de } \\
\text { los criterios de } \\
\text { evaluación del } \\
\text { sílabo con los } \\
\text { coordinadores } \\
\text { de curso, para } \\
\text { determinar el } \\
\text { listado de rubros } \\
\text { de evaluación que } \\
\text { deben tomarse en } \\
\text { cuenta. }\end{array}$ & $\begin{array}{l}\text { Es necesario } \\
\text { que los criterios } \\
\text { de evaluación } \\
\text { sean acordes y } \\
\text { orientados al logro } \\
\text { de competencias. }\end{array}$ \\
\hline 2 & $\begin{array}{l}\text { Los estudiantes } \\
\text { no reciben } \\
\text { información } \\
\text { de evaluación } \\
\text { continua / } \\
\text { medio ciclo } \\
\text { por parte } \\
\text { de algunos } \\
\text { docentes. }\end{array}$ & 1 & 3 & 3 & $\begin{array}{l}\text { - Sancionar a } \\
\text { los docentes que } \\
\text { incurren en esta } \\
\text { falta. } \\
\text { - Reprogramación de } \\
\text { la evaluación de ser } \\
\text { necesario. }\end{array}$ & $\begin{array}{l}\text { Los estudiantes } \\
\text { necesitan la } \\
\text { información } \\
\text { requerida para } \\
\text { desarrollar las } \\
\text { actividades de } \\
\text { evaluación con } \\
\text { normalidad. }\end{array}$ \\
\hline 3 & $\begin{array}{l}\text { Los resultados } \\
\text { de las } \\
\text { evaluaciones, } \\
\text { trabajos, } \\
\text { prácticas } \\
\text { no son } \\
\text { comunicados } \\
\text { oportunamente } \\
\text { por el docente } \\
\text { a cargo. }\end{array}$ & 1 & 3 & 3 & $\begin{array}{l}\text { - Realizar reuniones } \\
\text { para sensibilizar a } \\
\text { los docentes sobre } \\
\text { la necesidad de } \\
\text { entregar resultados } \\
\text { de evaluaciones } \\
\text { oportunamente. } \\
\text { - Sancionar a } \\
\text { los docentes que } \\
\text { incurran en esta } \\
\text { falta, para reforzar } \\
\text { este deber que tienen } \\
\text { los docentes frente } \\
\text { al derecho de los } \\
\text { alumnos de conocer } \\
\text { los resultados de sus } \\
\text { evaluaciones. }\end{array}$ & $\begin{array}{l}\text { Los estudiantes } \\
\text { deben reconocer } \\
\text { las fortalezas y } \\
\text { debilidades que se } \\
\text { les presenta en el } \\
\text { aprendizaje de cada } \\
\text { asignatura y para } \\
\text { ello debe visualizar } \\
\text { el qué, y cómo hizo } \\
\text { sus tareas, trabajos, } \\
\text { cualquier entrega } \\
\text { que demande una } \\
\text { nota cualitativa o } \\
\text { cuantitativa. }\end{array}$ \\
\hline
\end{tabular}




\section{Conclusiones}

La presente investigación logró la identificación de los procesos, las caracterizaciones y el análisis de riesgos, estos son aspectos fundamentales en la Facultad porque se tienen definidos los procesos y esto facilitará una mejora en la calidad del servicio educativo.

La caracterización de los procesos es importante dado que permite visualizar los aspectos críticos en la formulación de un proceso.

Un análisis de riesgos permite a la institución prever y mitigar los fallos, y las no conformidades (no cumplir con un requisito de la norma ISO) a fin de tomar acciones preventivas y establecer qué y quién las va a realizar, en caso de darse el riesgo.

La identificación de procesos, las caracterizaciones y el análisis de riesgo, facilitan la implementación de un SGC en la universidad que le permitirá organizar el trabajo con miras a la acreditación.

\section{REFERENCIAS}

Agudelo, L. \& Escobar, J. (2010). Gestión por procesos. Medellín: ICONTEC.

Bermeo, C. (2011). Evaluación de la calidad de la educación universitaria. Modelos vigentes. Revista EDUCACIÓN UNIFÉ, XVII, 36-45. Recuperado de: https://doi. org/10.33539/educacion.2011.n17.1441

Dolzhanskyy, A. M., Proydak, Y. S. \& Revenko, O. O. (2016). Application of analysis and risks assessment methods for quality assurance at the university. Science and Innovation, 12(5), 5-12. Recuperado de:

\section{Recomendaciones}

Es necesario socializar la información generada en esta investigación con las autoridades de la Institución de Educación Superior (Decano, Directores Académicos) para liderar el proceso de diseño e implementación del SGC, puesto que el tema de la calidad es una responsabilidad de todos los integrantes de la institución, partiendo de las autoridades.

Se recomienda realizar las caracterizaciones de todos los procesos estratégicos, principales y de soporte para complementar el proceso de la implementación del SGC. Además, realizar el análisis de riesgos de todos los procesos estratégicos, principales y de soporte para prevenir los fallos y las no conformidades.

La universidad debe implementar los procesos y los mecanismos de seguimiento necesarios (como auditorías internas, revisión por la dirección, entre otros) para una evaluación objetiva de su SGC y proponer planes de mejora respecto a las brechas detectadas.

https://doi.org/https://doi.org/10.15407/ scine12.05.005

Hernández, H., Martínez, D. \& Rodríguez, J. (2017). Gestión de la calidad aplicada en el mejoramiento del sector universitario. Espacios, 38(20), 29-33.

Illescas, Cabezas \& Diener (2006). Calidad en la formación de profesionales. Revista educación y Ciencias de la salud, 3(1), 1417. Recuperado de: http://www2.udec.cl/ ofem/recs/anteriores/vol312006/art3106b. pdf 
International Organization for Standarization (2018). Norma ISO 21001:2018 para las organizaciones educativas. Global STD. Recuperado de: https:/www.globalstd.com/ networks/blog/iso-21001-2018-para-lasorganizaciones-educativas

International Organization for Standarization (2015). Norma ISO 9001:2015. Sistema de Gestión de la Calidad - Requisitos. Instituto para la Calidad de la Pontificia Universidad Católica del Perú. Lima, Perú. 2018.

International Organization for Standarization (2005). Norma ISO 9000. Sistemas de Gestión de la Calidad - fundamentos y vocabulario. Instituto para la Calidad de la Pontificia Universidad Católica del Perú. Lima, Perú. 2018.

Ministerio de Educación del Perú (2018). Manual de Procedimientos. Recuperado de: http://www.minedu.gob.pe/transparencia/ pdf/manual_procedimientos/mapa_de_ procesos_minedu.pdf

Negrete, K. P., Jenyffer Yépez, E. M. O., NaranjoToro, M. \& Caraguay-Procel, J. (2020). Quality at the University Based on Process Management: Design and Implementation of the Quality Management System Under ISO 9001: 2015 Standard Applied to the Industrial Engineering Degree of the Northern Technical University. Advances in Intelligent Systems and Computing, 1110 AISC, 183-192.

Ortiz, P. (2008). Educación y formación de la personalidad. Lima: Fondo editorial de la Universidad de Ciencias y Humanidades.

Ruiz, J. (2012). Metodología de la investigación cualitativa. Lima: Serie Ciencias Sociales.

Sistema Nacional de Evaluación, Acreditación y Certificación de la Calidad Educativa
(2016). Modelo de Acreditación para Programas de Estudios de Educación Superior Universitaria. Lima: SINEACE.

Sistema Nacional de Evaluación, Acreditación y Certificación de la Calidad Educativa (2017). Modelo de Acreditación para Institucional. Lima: SINEACE.

Sistema Nacional de Evaluación, Acreditación y Certificación de la Calidad Educativa (2018). Acreditación. Lima: Ministerio de Educación. Recuperado de: https://www. sineace.gob.pe/acreditacion/

Consejo de Evaluación, Acreditación y Certificación de la Calidad de la Educación Superior Universitaria - CONEAU (2009). Guía para la acreditación de carreras profesionales universitarias del CONEAU. Lima - Perú: Ministerio de Educación.

Universidad Nacional Agraria La Molina (2018). Información Institucional de la Universidad Nacional Agraria La Molina. Recuperado de: http://www.lamolina.edu.pe/

Yamada, G., Castro, J., Bacigalupo, J. \& Valverde, L. (2013). Mayor acceso con menor calidad en la educación superior: algunas evidencias desde las habilidades de los estudiantes. Apuntes. Revista de Ciencias Sociales, 40(72), 7-32. Recuperado de: https://doi.org/https://doi.org/10.21678/ apuntes.72.673

Yamada, G. (2013). Calidad y acreditación de la educación superior: retos urgentes para el Perú. Lima: Universidad del Pacífico y CONEAU.

Zaher, B. H. (2020). Improving the Quality of Educational Services Using Quality Function Deployment: A Field Study on Syrian Universities. The Scientific Journal of King Faisal University, 21(2), 241-246. 